\title{
Correlation of survival length after pancreaticoduodenectomy for pancreatic head adenocarcinoma depending on tumor characteristics detected by means of computed tomography and resection margins status
}

\author{
V. PROCHAZKA ${ }^{1}$, J. HLAVSA ${ }^{1}$, L. KUNOVSKY ${ }^{1,2, *}$, M. FARKASOVA ${ }^{1}$, M. POTRUSIL ${ }^{1}$, T. ANDRASINA ${ }^{3}$, A. LITAVCOVA ${ }^{3}$, J. MAZANEC ${ }^{4}$, J. DOLINA ${ }^{2}$, \\ L. OSTRIZKOVA ${ }^{5}$, M. EID ${ }^{5}$, P. KOVALCIKOVA ${ }^{6}$, T. PAVLIK ${ }^{6}$, Z. KALA ${ }^{1}$
}

\begin{abstract}
${ }^{1}$ Department of Surgery, University Hospital Brno, Faculty of Medicine, Masaryk University, Brno, Czech Republic; ${ }^{2}$ Department of Gastroenterology and Internal Medicine, University Hospital Brno, Faculty of Medicine, Masaryk University, Brno, Czech Republic; ${ }^{3}$ Department of Radiology and Nuclear Medicine, University Hospital Brno, Faculty of Medicine, Masaryk University, Brno, Czech Republic; ${ }^{4}$ Department of Pathology, University Hospital Brno, Faculty of Medicine, Masaryk University, Brno, Czech Republic; ${ }^{5}$ Department of Hematology, Oncology and Internal Medicine, University Hospital Brno, Faculty of Medicine, Masaryk University, Brno, Czech Republic; ${ }^{6}$ Institute of Biostatistics and Analyses, Faculty of Medicine, Masaryk University, Brno, Czech Republic
\end{abstract}

${ }^{*}$ Correspondence: lumir.kunovsky@gmail.com

Received September 23, 2019 / Accepted February 19, 2020

\begin{abstract}
Pancreatic carcinoma is an aggressive tumor with a grim prognosis. Accurate staging is essential for indicating surgery in patients with borderline resectable tumors. This paper examines the correlation between pre-operation characteristics of tumors found on CT, infiltration of individual resection margins as confirmed by a pathologist, and the survival of patients with resectable pancreatic head ductal adenocarcinoma. This prospective cohort study involved patients operated on for pancreatic head adenocarcinoma, which was clearly resectable based on the staging CT and intraoperative observation between 2011-2014. Only patients without postoperative complications who underwent adjuvant chemotherapy were analyzed. Seventy-nine patients were assessed, of which 16 (20.3\%) had R0 resection and 63 (79.7\%) had R1 resection. Patients with R1 results had up to 2.7 times higher risk of death than patients with R0 resection. We found a trend towards shorter survival associated with a closer relationship of the tumor to the superior mesenteric vein/portal vein (SMV/PV) wall in the pre-operation CT examination. Patients with a tumor interface between the vein wall of up to $180^{\circ}$ circumference had up to 1.97 times higher risk of death than patients without $(\mathrm{p}=0.131)$. The results of our work confirmed that in our center, even surgically treated, clearly resectable pancreatic head tumors still have a high occurrence of positive surgical margins (R1 resection) and that tumors with R1 resection had statistically significantly reduced survival compared to R0 resection. A trend for shorter overall survival was found after tumor resection depending on the increasing interface between the tumor and the SMV/PV wall, but this result was not statistically significant.
\end{abstract}

Key words: pancreatic cancer, pancreaticoduodenectomy, overall survival, resection margin, computed tomography, surgery

Pancreatic ductal adenocarcinoma (PDAC) is one of the most common tumors of the digestive tract and the seventh most frequent cause of death from oncological diseases [1]. Resection is the only potentially curative therapeutic modality. At the time of diagnosis, $80 \%$ of all patients are already in the stage of an advanced, surgically incurable disease [2]. The survival median published for patients who underwent resection surgery is adverse, ranging between 15 and 23 months [3]. In 1994, Allema et al. published a cohort study of 20 patients who underwent a pancreaticoduodenectomy (PD) combined with SMV or PV resection; the survival of these patients was the same as after standard PD [4]. Tseng et al. published a paper in which the survival of 110 patients with vascular resection combined with PD was compared to 181 patients with standard PD. The survival was three months shorter in the group with vascular resection [5].

With tumors localized in the head of the pancreas, it is important to consider their interface with the superior mesenteric vein (SMV), the portal vein (PV), the superior mesenteric artery (SMA), and the hepatic artery (HA). Depending on this interface, the tumors are defined as either resectable, borderline resectable (BRPC), or non-resectable pancreatic cancer [6]. Findings on the staging CT and acknowledged criteria for BRPC include: an interface between the tumor and 
SMV or PV of $180^{\circ}$ or greater, with the possibility of vessel resection, encasement of gastroduodenal artery continuing to the HA without progression towards the coeliac axis, and SMA abutment $<180^{\circ}$ of its circumference [7]. Tumors with a smaller extent of the interface towards the vascular wall are considered resectable, and these patients are indicated for primary resection [8].

Patients with microscopically positive resection margins (R1) have significantly shorter survival than patients without the presence of tumor in the resection margins (R0). The survival of patients with macroscopically apparent tumor residue (R2) is comparable to patients who have not been operated on at all $[9,10]$. Verbeke et al. published a study on the assessment of seven resection margins in 2006 [11].

Therefore, the purpose of this paper is to study the possible correlation between the pre-operation characteristics of the tumor found on CT, the infiltration of the individual resection margins as confirmed by a pathologist, and the survival of patients with resectable PDAC of the head.

For the purposes of our work, the main assessment criterion is the interface between the tumor and the SMV/PV wall. A simple classification was described by Ishikawa et al. in 1992 [12]. Tumors are resectable if they are graded 1 to 3 (1/ no contact between tumor and SMV/PV wall, 2/ smooth shift narrowing, 3/ unilateral narrowing). With a lesion of a greater extent, these tumors are BRPC or non-resectable. In our work, we will focus on the assessment of whether there are any definable prognostic differences following resection within the group of resectable pancreatic head carcinomas.

\section{Patients and methods}

Patients. Patients operated on for pancreatic head carcinoma at the Department of Surgery of the University Hospital Brno between 2011 and 2014 were included in the study. Only patients with clearly resectable tumors were enrolled. The study was designed as a prospective cohort study. Resectability was assessed upon pre-operation CT as well as during the operation. Resectable tumors were those without any interface with SMA or HA on the pre-operation CT and during the operation, and where no SMV or PV resection was required. The size of our cohort was also determined by a strict selection. Only patients without any serious postoperation complications who could undergo post-operation adjuvant oncological treatment were included: in cases of the R0 resection it was chemotherapy with gemcitabine, in cases of $\mathrm{R} 1$ resection it was a combination of radiotherapy and gemcitabine chemotherapy. The patients were observed for a minimum of 36 months.

Surgical procedure. All of the patients underwent either a pylorus preserving pancreaticoduodenectomy (PD) or total pancreatectomy (TP). TP was indicated during surgery in cases where the tumorous infiltration reached into the body of the pancreas and where the soft pancreas did not allow for a safe anastomosis on the pancreatic body. All of the surgical procedures were performed by three surgeons who had each previously been involved in more than 100 resections of pancreatic head tumors.

The methodology of CT findings assessment. The following parameters were assessed: tumor margins, the relation of the tumor to SMV/PV wall, tumor size.

Tumor margins: In the late arterial and portal venous phase of the examination (15 and 55 seconds after bolus tracking, respectively) we assessed the margin of the tumor against a healthy gland. A site lesion that demonstrated a significant difference in density compared to the pancreatic parenchyma (more than $25 \mathrm{HU}$ - Hounsfield units) in one or both post contrast data sets and which had defined contoured edges was categorised as having a clearly defined margin (Figure 1A). A lesion with a blurred contour and a lower difference compared to the density of the gland (10-25 HU) was categorized in two degrees as poorly defined (Figure 1B). A lesion with margins, which were impossible to define and where the presence of tumor was assessed upon indirect signs only (loss of lobulated structure in the pancreas, dilation of the pancreatic duct, or ductus choledochus), was categorized as undefined (Figure 1C), i.e. a lesion with a visible infiltrative growth (contrast difference less than $10 \mathrm{HU})$.

Interface between the tumor and SMV/PV wall: The interface between the tumor and the wall of the PV or the SMV was determined upon axial sections and upon multiplanar reconstructions of the portal venous phase of the CT examination. We assessed the existence of a fat dense strip around the vessel, and the distance between the tumor and the PV stem. The first degree represented a finding without any interface between the tumor and the vascular wall. If the fat strip between the vessel and the lesion was absent and there was contact between the vessel and the tumor up to $90^{\circ}$ of the vessel's circumference, the relation was evaluated as insignificant contact. If the contact of the tumor ranged between $90-180^{\circ}$ of the vessel's circumference, it was evaluated as significant contact.

Tumor size: The size of the tumor was measured in the late arterial phase or portal venous phase of a contrast CT examination (dependent on higher contrast difference).

The methodology for assessing the pathological findings. The Leeds protocol [11] currently represents the recommended procedure for the pathological processing of tumor tissue resected from the pancreatic head. This procedure of processing is based on the assessment of the commonly examined edges of the resected tissue in the pancreas neck region, the choledochus and both ends of the duodenum, a thorough examination of the radial (circumferential) edges of the resected tissue and the surfaces on the anterior and posterior side of the pancreatic head, and along the superior mesenteric vessels. The ventral and the dorsal line are defined as the mobilization lines, other lines are defined as transsection lines. 
Statistical assessment methodology. The basic characteristics of patients with PDAC based on the radicality of the resection surgery upon assessing the individual resection lines have been summarized using absolute and relative counts and compared by means of the Fisher Exact Test (categorical variables). Continuous characteristics were summarized by means of average, standard deviation (SD), median, minimum and maximum, and compared by means of the Mann-Whitney test.

The relation between the basic characteristics of the patient, the results of the radiology examination, and the radicality of the pancreatic resection was assessed using unidimensional logistic regression.

The positivity of individual resection lines by the relation of the tumor to the vein wall on the CT image prior to the pancreatic resection was summarized using absolute and relative counts and compared by means of the Fisher exact test.

The overall survival of patients with PDAC according to their individual characteristics, radiological results, and the radicality of the resection were evaluated by means of the Kaplan-Meier method. A two-tailed p-value $<0.05$ was considered significant for all analyses.

\section{Results}

Basic characteristics of the patients. A total of 79 patients were assessed, 37 of whom were over 65 years old. In 16 patients $(20.3 \%)$, the histological examination results stated R0 resection and in 63 (79.7\%), R1 resection. In total, $67 \mathrm{PD}$ and $12 \mathrm{TP}$ were performed. The majority of the patients had a T3 tumor (96.2\%) and lymph node positivity was found in $84.8 \%$ of patients operated on. Table 1 summarizes and compares the basic characteristics of the patients operated on for PDAC.

Table 2 presents the results of the histological examination with the assessment of individual resection lines. The most frequently recorded positivity was that of the ventral resection line (in $57.1 \%$ from the total number of $\mathrm{R} 1$ resections) and the resection line between SMV/PV (in 55.6\% patients from the total number of R1 resections). Perivascular infiltration of the tumor was found in $36.7 \%$ and perineural infiltration was found in $94.9 \%$ of cases. During the assessment of the infiltration of the individual resection lines in R1 resections, 12 patients of 63 (19\%) had negative transsection lines and only their ventral or dorsal resection lines were positive.

During the assessment of the pre-operation CT findings, the median size of the tumors found was $27 \mathrm{~mm}$. The most frequently found tumor margin character was infiltrative growth without a clearly defined lesion - in $36.7 \%$ of cases. No correlation was found between the tumor's interface with the SMV/PV wall on CT and the assessment of resection radicality performed by the pathologist. The results of the
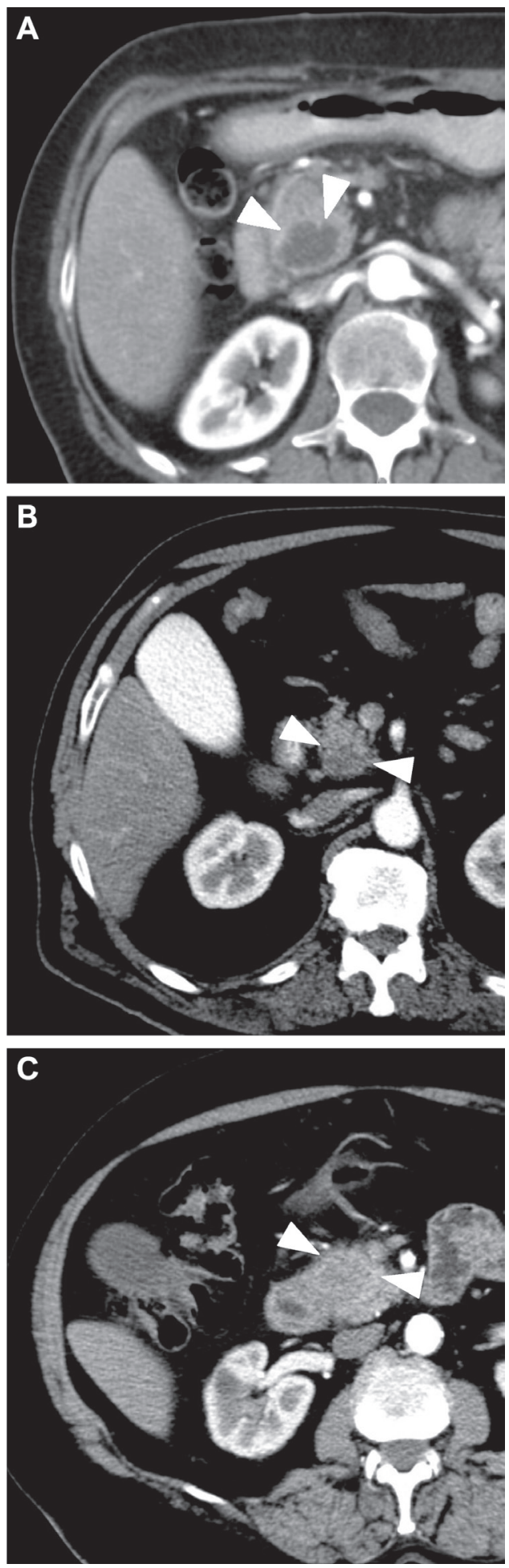

Figure 1. Assessment of tumor margin using CT. A) A lesion with a significant difference in density when compared to the pancreatic parenchyma and well defined contoured edges, categorized as a clearly defined margin. B) A lesion with a blurred contour and lower difference compared to the density of the gland (10-25 HU), categorized as poorly defined. C) A lesion with margins impossible to define and where the presence of the tumor had been assessed upon indirect signs only categorized as undefined. 
radiological examinations and the relation to R0/R1 resections are presented in Table 3.

Assessment of the relation between pancreatic tumor and SMV/PV by CT and radicality of resection. Table 4 summarizes and compares both the number and positivity of the individual resection lines in patients with PDAC depending on the relationship between the tumor and the SMV/PV wall upon CT image. Among those patients assessed based on the tumor interface with the vein wall (no relation, insignificant contact, and contact up to $180^{\circ}$ of circumference), a statistically significant difference was found only in the positivity of the resection line on the body of the pancreas (assessed only in patients who underwent PD). Patients with an interface between the tumor and vein wall of up to a $180^{\circ}$ circumference showed a more frequent positivity of this resection line than patients without any interface between the tumor and the vein wall, or with just insignificant contact. In Group I (no contact), the SMV/PV resection line was positive in $31.6 \%$ of cases, in Group II (insignificant contact), it was $56.7 \%$ and in Group III (contact up to $180^{\circ}$ circumference), a positivity was found in $54.5 \%$ of patients.
However, these differences did not reach the threshold of statistical significance.

Table 5 compares the number of affected lines in patients with R1 resection only. No statistically significant difference in the number of affected resection lines was found between the patients without an interface between the tumor and vein wall, and those with a more significant interface between the tumor and the SMV/PV wall. More than $70 \%$ of patients with $\mathrm{R} 1$ resection had two or more positive resection lines, regardless of their CT-determined interface between the tumor and the SMV/PV wall. One or more other positive resection lines were found in $91.4 \%$ of patients with a positive SMV/PV line.

Assessment of overall survival based on the patient characteristics, pre-operation radiology results, and the radicality of the resection. Table 6 presents the results of unidimensional Cox regression models, quantifying the relationship between overall survival after pancreas resection and the patient's individual characteristics, pre-operation radiology results, and the results of the histological examination after the operation. The hazard ratio expresses how many times higher is the hazard of death for the risk category when

Table 1. Summary of the basic characteristics of patients who underwent surgery for pancreatic ductal adenocarcinoma.

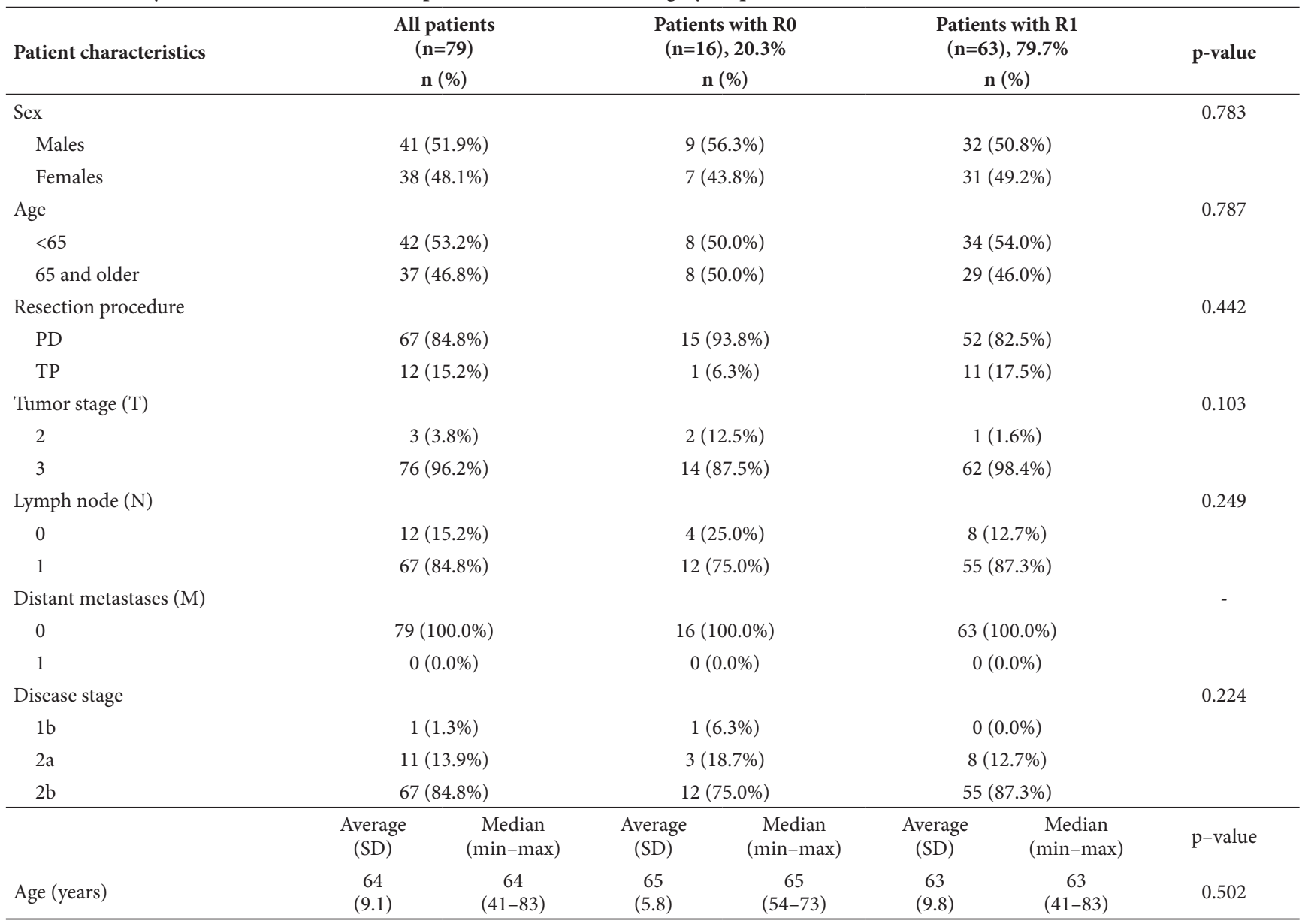

PD - pancreaticoduodenectomy, TP - total pancreatectomy 
Table 2. Summary of the histological examination results after pancreas resection in patients with pancreatic ductal adenocarcinoma by the radicality of the resection based on the Leeds protocol.

\begin{tabular}{|c|c|c|c|c|}
\hline Histological examination results & $\begin{array}{c}\text { All patients }(\mathrm{n}=79) \\
\mathrm{n}(\%)\end{array}$ & $\begin{array}{c}\text { Patients with R0 (n=16) } \\
\text { n (\%) }\end{array}$ & $\begin{array}{c}\text { Patients with R1 (n=63) } \\
\text { n (\%) }\end{array}$ & p-value \\
\hline Infiltration to the peripancreatic soft tissue & & & & 0.005 \\
\hline No & $5(6.3 \%)$ & $4(25.0 \%)$ & $1(1.6 \%)$ & \\
\hline Yes & $74(93.7 \%)$ & $12(75.0 \%)$ & $62(98.4 \%)$ & \\
\hline Perivascular tumor infiltration & & & & 1.000 \\
\hline No & $50(63.3 \%)$ & $10(62.5 \%)$ & $40(63.5 \%)$ & \\
\hline Yes & $29(36.7 \%)$ & $6(37.5 \%)$ & $23(36.5 \%)$ & \\
\hline Perineural tumor infiltration & & & & 1.000 \\
\hline No & $4(5.1 \%)$ & $1(6.3 \%)$ & $3(4.8 \%)$ & \\
\hline Yes & $75(94.9 \%)$ & $15(93.8 \%)$ & $60(95.2 \%)$ & \\
\hline Ventral - anterior margin & & & & - \\
\hline Negative & $43(54.4 \%)$ & $16(100.0 \%)$ & $27(42.9 \%)$ & \\
\hline Positive & $36(45.6 \%)$ & $0(0.0 \%)$ & $36(57.1 \%)$ & \\
\hline Dorsal - posterior margin & & & & - \\
\hline Negative & $52(65.8 \%)$ & $16(100.0 \%)$ & $36(57.1 \%)$ & \\
\hline Positive & $27(34.2 \%)$ & $0(0.0 \%)$ & $27(42.9 \%)$ & \\
\hline Arterial line & & & & - \\
\hline Negative & $53(67.1 \%)$ & $16(100.0 \%)$ & $37(58.7 \%)$ & \\
\hline Positive & $26(32.9 \%)$ & $0(0.0 \%)$ & $26(41.3 \%)$ & \\
\hline Vein wall line & & & & - \\
\hline Negative & $44(55.7 \%)$ & $16(100.0 \%)$ & $28(44.4 \%)$ & \\
\hline Positive & $35(44.3 \%)$ & $0(0.0 \%)$ & $35(55.6 \%)$ & \\
\hline Pancreas body line & & & & - \\
\hline Negative & $59(74.7 \%)$ & $15(93.8 \%)$ & $44(69.8 \%)$ & \\
\hline Positive & $8(10.1 \%)$ & $0(0.0 \%)$ & $8(12.7 \%)$ & \\
\hline Not assessed & $12(15.2 \%)$ & $1(6.3 \%)$ & $11(17.5 \%)$ & \\
\hline Bile duct line & & & & - \\
\hline Negative & $79(100.0 \%)$ & $16(100.0 \%)$ & $63(100.0 \%)$ & \\
\hline Positive & $0(0.0 \%)$ & $0(0.0 \%)$ & $0(0.0 \%)$ & \\
\hline Duodenum line & & & & - \\
\hline Negative & $78(98.7 \%)$ & $16(100.0 \%)$ & $62(98.4 \%)$ & \\
\hline Positive & $1(1.3 \%)$ & $0(0.0 \%)$ & $1(1.6 \%)$ & \\
\hline Radicality by the positivity of the transsection lines & & & & - \\
\hline R0 & $28(35.4 \%)$ & $16(100.0 \%)$ & $12(19.0 \%)$ & \\
\hline $\mathrm{R} 1$ & $51(64.6 \%)$ & $0(0.0 \%)$ & $51(81.0 \%)$ & \\
\hline
\end{tabular}

Table 3. Summary of the radiological examination results of patients with pancreatic ductal adenocarcinoma and comparison with resection radicality based on the Leeds protocol.

\begin{tabular}{|c|c|c|c|c|c|c|c|}
\hline $\begin{array}{l}\text { Radiological examination results } \\
\text { (upon CT image) }\end{array}$ & \multicolumn{2}{|c|}{$\begin{array}{c}\text { All patients }(\mathrm{n}=79) \\
\mathbf{n}(\%)\end{array}$} & \multicolumn{2}{|c|}{$\begin{array}{l}\text { Patients with R0 }(\mathrm{n}=16) \\
\text { n (\%) }\end{array}$} & \multicolumn{2}{|c|}{$\begin{array}{l}\text { Patients with R1 }(\mathrm{n}=63) \\
\text { n (\%) }\end{array}$} & p-value \\
\hline Tumor margin & & & & & & & 0.606 \\
\hline Clearly defined & \multicolumn{2}{|c|}{$23(29.1 \%)$} & \multicolumn{2}{|c|}{$3(18.8 \%)$} & \multicolumn{2}{|c|}{$20(31.8 \%)$} & \\
\hline Poorly defined & \multicolumn{2}{|c|}{$27(34.2 \%)$} & \multicolumn{2}{|c|}{$6(37.5 \%)$} & \multicolumn{2}{|c|}{$21(33.3 \%)$} & \\
\hline Infiltrative growth & \multicolumn{2}{|c|}{$29(36.7 \%)$} & \multicolumn{2}{|c|}{$7(43.8 \%)$} & \multicolumn{2}{|c|}{$22(34.9 \%)$} & \\
\hline Tumor interface with vein wall & & & & & & & 0.640 \\
\hline No interface & \multicolumn{2}{|c|}{$38(48.1 \%)$} & \multicolumn{2}{|c|}{$9(56.3 \%)$} & \multicolumn{2}{|c|}{$29(46.0 \%)$} & \\
\hline Insignificant contact & \multicolumn{2}{|c|}{$30(38.0 \%)$} & \multicolumn{2}{|c|}{$6(37.5 \%)$} & \multicolumn{2}{|c|}{$24(38.1 \%)$} & \\
\hline Contact up to $180^{\circ}$ of circumference & \multicolumn{2}{|c|}{$11(13.9 \%)$} & \multicolumn{2}{|c|}{$1(6.3 \%)$} & \multicolumn{2}{|c|}{$10(15.9 \%)$} & \\
\hline & $\begin{array}{c}\text { Average } \\
\text { (SD) }\end{array}$ & $\begin{array}{c}\text { Median } \\
(\min -\max )\end{array}$ & $\begin{array}{l}\text { Average } \\
\text { (SD) }\end{array}$ & $\begin{array}{c}\text { Median } \\
(\min -\max )\end{array}$ & $\begin{array}{l}\text { Average } \\
\text { (SD) }\end{array}$ & $\begin{array}{c}\text { Median } \\
(\min -\max )\end{array}$ & \\
\hline $\begin{array}{l}\text { Tumor size } \\
\text { (in mm) }\end{array}$ & $\begin{array}{c}27 \\
(6.9)\end{array}$ & $\begin{array}{c}27 \\
(14-48)\end{array}$ & $\begin{array}{c}28 \\
(9.3)\end{array}$ & $\begin{array}{c}26 \\
(15-48)\end{array}$ & $\begin{array}{c}27 \\
(6.2)\end{array}$ & $\begin{array}{c}27 \\
(14-47)\end{array}$ & 0.641 \\
\hline
\end{tabular}


Table 4. Summary and comparison of the positivity of the individual resection lines in patients with pancreatic ductal adenocarcinoma based on the interface between the tumor and the vein wall as determined on the CT image.

\begin{tabular}{|c|c|c|c|c|}
\hline \multirow[b]{2}{*}{ Positivity of resection lines } & \multicolumn{3}{|c|}{ Patients by tumor interface with the vein wall on CT image } & \multirow[b]{2}{*}{ p-value } \\
\hline & $\begin{array}{c}\text { No interface }(\mathbf{n}=38) \\
\mathbf{n}(\%)\end{array}$ & $\begin{array}{l}\text { Insignificant contact }(\mathrm{n}=30) \\
\mathrm{n}(\%)\end{array}$ & $\begin{array}{c}\text { Interface up to } 180^{\circ} \text { of } \\
\text { circumference }(n=11) \\
n(\%)\end{array}$ & \\
\hline Ventral margin & & & & 0.501 \\
\hline Negative & $19(50.0 \%)$ & $19(63.3 \%)$ & $5(45.5 \%)$ & \\
\hline Positive & $19(50.0 \%)$ & $11(36.7 \%)$ & $6(54.5 \%)$ & \\
\hline Dorsal margin & & & & 0.096 \\
\hline Negative & $21(55.3 \%)$ & $24(80.0 \%)$ & $7(63.6 \%)$ & \\
\hline Positive & $17(44.7 \%)$ & $6(20.0 \%)$ & $4(36.4 \%)$ & \\
\hline Arterial line & & & & 0.611 \\
\hline Negative & $27(71.1 \%)$ & $20(66.7 \%)$ & $6(54.5 \%)$ & \\
\hline Positive & $11(28.9 \%)$ & $10(33.3 \%)$ & $5(45.5 \%)$ & \\
\hline Vein wall line & & & & 0.093 \\
\hline Negative & $26(68.4 \%)$ & $13(43.3 \%)$ & $5(45.5 \%)$ & \\
\hline Positive & $12(31.6 \%)$ & $17(56.7 \%)$ & $6(54.5 \%)$ & \\
\hline Pancreas body line & & & & $0.042^{*}$ \\
\hline Negative & $28(73.7 \%)$ & $24(80.0 \%)$ & $7(63.6 \%)$ & \\
\hline Positive & $2(5.3 \%)$ & $2(6.7 \%)$ & $4(36.4 \%)$ & \\
\hline Not assessed & $8(21.1 \%)$ & $4(13.3 \%)$ & $0(0.0 \%)$ & \\
\hline Bile duct line & & & & - \\
\hline Negative & $38(100.0 \%)$ & $30(100.0 \%)$ & $11(100.0 \%)$ & \\
\hline Positive & $0(0.0 \%)$ & $0(0.0 \%)$ & $0(0.0 \%)$ & \\
\hline Duodenum line & & & & 0.519 \\
\hline Negative & $38(100.0 \%)$ & $29(96.7 \%)$ & $11(100.0 \%)$ & \\
\hline Positive & $0(0.0 \%)$ & $1(3.3 \%)$ & $0(0.0 \%)$ & \\
\hline Affected lines total & & & & 0.896 \\
\hline 0 & $9(23.7 \%)$ & $6(20.0 \%)$ & $1(9.1 \%)$ & \\
\hline 1 & $8(21.1 \%)$ & $7(23.3 \%)$ & $2(18.2 \%)$ & \\
\hline 2 and more & $21(55.3 \%)$ & $17(56.7 \%)$ & $8(72.7 \%)$ & \\
\hline
\end{tabular}

*The comparison relates only to cases with assessed pancreas body line (i.e. patients who underwent pancreaticoduodenectomy).

Table 5. Comparison of the number of affected resection lines based on the Leeds protocol in patients with R1 resection by the interface of the tumor and the vein wall.

\begin{tabular}{lccc}
\hline & \multicolumn{2}{c}{ Patients by tumor interface with the vein wall on CT image } \\
\cline { 2 - 4 } Affected lines total & $\begin{array}{c}\text { No interface }(\mathbf{n}=\mathbf{2 9}) \\
\mathbf{n}(\%)\end{array}$ & $\begin{array}{c}\text { Insignificant contact (n=24) } \\
\mathbf{n}(\%)\end{array}$ & $\begin{array}{c}\text { Interface up to 180 } \\
\text { circumference }(\mathbf{n}=\mathbf{1 0}) \\
\mathbf{n}(\%)\end{array}$ \\
\hline 1 affected resection line & $8(27.6 \%)$ & $7(29.2 \%)$ & $2(20.0 \%)$ \\
2 and more affected resection lines & $21(72.4 \%)$ & $17(70.8 \%)$ & $8(80.0 \%)$ \\
\hline
\end{tabular}

compared to the reference one. The Kaplan-Meier survival curves according to the individual parameters are shown in Figure 2.

A statistically significant difference was shown in the overall survival from the pancreas resection for the parameters of resection radicality assessment when using the Leeds Protocol, as well as for the positivity in the transsectional lines. Patients with $\mathrm{R} 1$ resection lines assessed using the
Leeds protocol have up to 2.7 times higher risk of death than patients with a negative result of R0 (HR: 2.68; 95\% CI for HR: 1.11-6.37). Patients with R1 resection assessed by the positivity of the transsectional lines have a two times higher risk of death than patients with R0 resection (HR: 2.00, 95\% CI for HR: 1.02-3.86).

Patients with a tumor to vein wall interface of up to $180^{\circ}$ of circumference had a greater risk of death compared to 
Table 6. Survival median and evaluation of the relationship between the overall survival of patients with pancreatic ductal adenocarcinoma and the individual observed parameters by means of unidimensional Cox regression models.

\begin{tabular}{|c|c|c|c|c|}
\hline & $n$ & Survival median & Unidimensional Cox regression model & 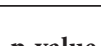 \\
\hline & $\mathrm{n}$ & Months $(95 \% \mathrm{CI})^{*}$ & HR (95\% CI) & p-value \\
\hline All patients & 79 & $23(18 ; 28)$ & - & - \\
\hline Sex & & & & \\
\hline Males & 41 & $23(14 ; 38)$ & $1.00(-)$ & - \\
\hline Females & 38 & $22(16 ; 31)$ & $0.97(0.54 ; 1.75)$ & 0.907 \\
\hline Age & & & & \\
\hline$<65$ & 42 & $23(18 ; 38)$ & $1.00(-)$ & - \\
\hline 65 and older & 37 & $19(13 ; 29)$ & $1.16(0.64 ; 2.10)$ & 0.619 \\
\hline Disease stage & & & & \\
\hline $2 \mathrm{a}$ & 11 & - & $1.00(-)$ & - \\
\hline $2 b$ & 67 & $22(16 ; 25)$ & $2.21(0.77 ; 6.09)$ & 0.133 \\
\hline Resection radicality ba & Leed & & & \\
\hline R0 & 16 & - & $1.00(-)$ & - \\
\hline R1 & 63 & $20(16 ; 24)$ & $2.68(1.11 ; 6.37)$ & 0.027 \\
\hline Resection radicality ba & posit & nal lines & & \\
\hline R0 & 28 & $38(19 ;-)$ & $1.00(-)$ & - \\
\hline R1 & 51 & $19(14 ; 23)$ & $2.00(1.02 ; 3.86)$ & 0.042 \\
\hline Tumor margin on CT & & & & \\
\hline Clearly & 23 & $22(14 ;-)$ & $1.00(-)$ & - \\
\hline Poorly & 27 & $17(11 ; 31)$ & $1.32(0.62 ; 2.77)$ & 0.467 \\
\hline Infiltrative growth & 29 & $25(14 ;-)$ & $0.87(0.42 ; 1.81)$ & 0.699 \\
\hline Tumor interface with $v$ & 11 on & & & \\
\hline No interface & 38 & $23(16 ; 38)$ & $1.00(-)$ & - \\
\hline Insignificant & 30 & $20(12 ; 31)$ & $1.13(0.60 ; 2.17)$ & 0.711 \\
\hline Up to $180^{\circ}$ & 11 & $19(7 ;-)$ & $1.97(0.80 ; 4.67)$ & 0.131 \\
\hline Tumor size on CT ima & & & & \\
\hline Increase by $10 \mathrm{~mm}$ & 79 & - & $1.15(0.76 ; 1.74)$ & 0.513 \\
\hline
\end{tabular}

${ }^{*}$ In some cases, the survival median and/or 95\% CI reliability could not be assessed for the survival median (noted -).

A
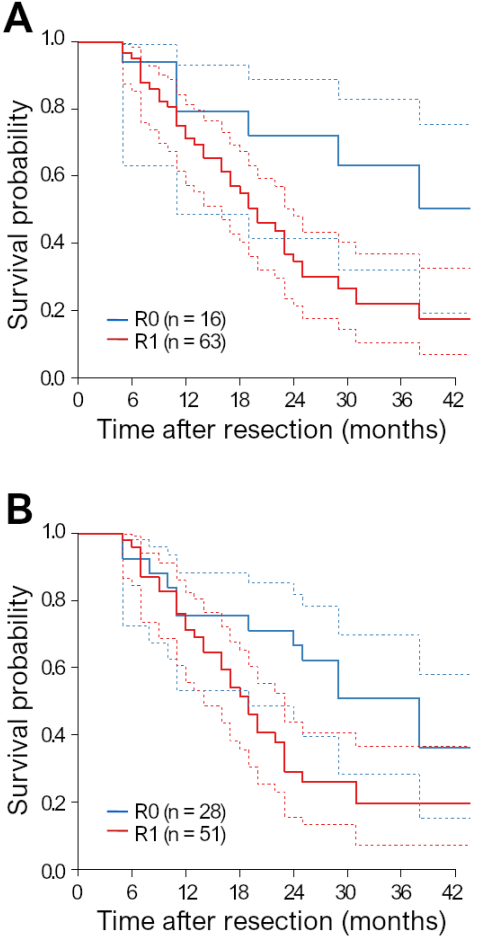
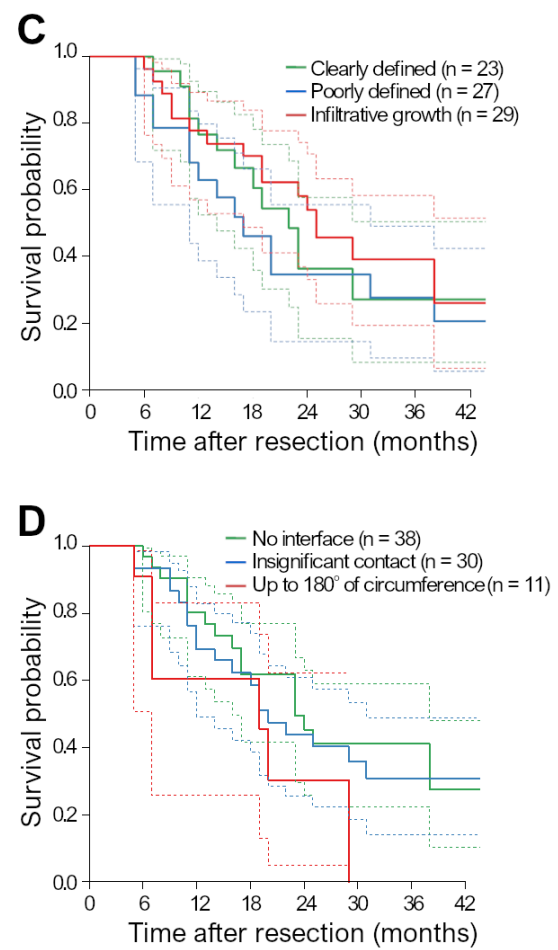

Figure 2. A - Radicality of the resection based on the Leeds protocol, B - Radicality of the resection based on the assessment of positivity of the transsectional lines, C - Tumor margin determined on CT image, D - Tumor interface with the vein wall on CT image. A and $B$ ) Kaplan-Meier estimation of the overall survival of patients with pancreatic carcinoma (full line) with 95\% CI (dashed line) based on the radicality of resection assessed by a pathologist. C and D) Kaplan-Meier estimate of the overall survival of patients with pancreatic carcinoma (full line) with $95 \%$ CI (dashed line) based on the results of the radiological examination before the operation. 
patients without a tumor interface with the vein wall, as determined on the CT image. A difference is suggested in the overall survival after pancreas resection, depending on the interface between the tumor and the vein wall, as determined on the CT. Patients with a tumor interface between the vein wall of up to $180^{\circ}$ of circumference may have up to 1.97 times higher risk of death than patients without a tumor interface with the vein wall determined on the CT image (HR: 1.97; 95\% CI for HR: 0.80-4.67). The survival median of patients without a tumor interface with the vein wall on the CT was 23 months, with smooth shift narrowing it was 20 months, and with the tumor in contact with the SMV/PV wall up to $180^{\circ}$ circumference, it was 19 months. The results, however, were not clearly statistically significant $(\mathrm{p}=0.131)$.

\section{Discussion}

The characterization of anatomical relations between the PDAC and the surrounding large vessels is a critical phase in the decision-making process regarding the overall strategy of the therapy and the surgical procedure. Staging examination methods relating to pancreatic tumors still focus on the assessment of the interface with the large vessels and the exclusion of distant metastases. In cases of an advanced local stage, pancreas resections can be performed together with resection or replacement of the SMP/PV affected by the tumor. According to recent studies, these procedures have a higher risk of postoperation complications and are followed by shorter survival than those without vessel resection [13]. A possible explanation is a more advanced stage and worse biological characteristics of the tumor if vascular structures are infiltrated. In our population, we found no difference in the percentage of R0 and $\mathrm{R} 1$ resections according to their interface with the SMV/ PV wall as determined on CT examination. On the other hand, we found a trend towards shorter survival associated with the increasing relation of the tumor to the vein wall.

When a pathologist examines the resected tissue, resection lines other than just SMV/PV are also assessed [14]. The aim of the examination methods should therefore ideally be to find out before the operation whether an $\mathrm{R} 0$ resection can be achieved on all lines, but the CT does not allow for this. It is possible to perform a cryobioptic examination of the resection lines and if they are tumor-positive, change the strategy of the operation and perform a more extensive procedure. In these cases, the contribution to survival improvement is questionable, as the study of Mathur et al. [15] and as well Dikman et al. [16] has shown. Patients with $\mathrm{R} 1$ resection determined preoperatively by cryobiopsy clearly have a worse prognosis than patients with $\mathrm{R} 0$ resection.

The effort to achieve an $\mathrm{R} 0$ procedure by the repeated resection of a previously positive line does not have an impact on survival improvement and therefore, according to Mathur et al., another extensive procedure does not make sense [15]. In our cohort, $73 \%$ of patients had two or more positive resection lines (R1 resection). Extending one of the resection lines does not change the fact that it is an $\mathrm{R} 1$ resection, which also relates to the potential resection of SMV/PV if its infiltration is present. In our cohort, SMV/PV positivity meant the more frequent presence of at least one other positive line, specifically in $91.4 \%$ of cases. This may explain the published poor survival of patients for whom vessel resection during PD was necessary [13].

There is a discussion in the literature on whether the positivity of all the resection lines has the same prognostic impact on post-operation survival. Jamieson et al. published a paper in which he divided the resection lines into mobilization (ventral and dorsal line) and transsection lines. Infiltration of the transsection lines showed a worse survival length prognosis in his population than the infiltration of the mobilization lines [17]. In a French study involving 150 patients, it was found that a positive dorsal margin did not influence the number of local recurrences, whereas in positive transsection margins this relationship had been proven [18]. One of the possible explanations relates to the development in the treatment of colorectal carcinoma where dissection according to embryonically established layers is a standard procedure nowadays [19]. Surgical liberation of the pancreas in the anterior and posterior pancreatic plane takes place within these layers and does not lead to the interruption of any lymphatic or vascular structures. The resection lines present between the adventitia of large vessels, pancreatic carcinoma, and lymphatic pathways are located in the area of the potential direction of the tumor invasion and therefore pose a greater risk of worse survival if found positive. In our cohort, we noted the most frequent positivity on the ventral resection line and the SMV/PV line. Only in 12 patients did we note a negative transsection line with positive mobilization lines.

Neoadjuvant therapy allows for the selection of patients with a rapid progression of the disease, while restaging eliminates the patients who would not benefit from the demanding resection therapy [20]. None of our patients met the criteria for the BRPC group and neoadjuvant therapy was not indicated. We noted $79.1 \%$ of R1 resections. The incidence of $\mathrm{R} 1$ resections in our population is similar to those in other publications [21].

Further studies are needed to verify the clinical impact of the individual resection lines' positivity. The literature demonstrates a consensus that $\mathrm{R} 1$ resection is a negative prognostic factor for longer survival, but the conclusions remain unequivocal concerning the methodology of histopathological examination of the resected tissue and the number of resection lines examined, as well as regarding the interpretation of the resection line's positivity. On a large patient cohort, Hartwig et al. proved that assessing R1 resection when the tumor was less than $1 \mathrm{~mm}$ away from the margin significantly influenced the accuracy of determining the prognosis of the patients [22]. In our work, such resection is therefore considered R1 even if some other departments would only consider direct infiltration of the assessed line by the tumor as $\mathrm{R} 1$ resection. 
The relationship of the pre-operation $\mathrm{CT}$ and the length of survival depending on the interface with SMV/PV had been studied earlier in locally advanced tumors. Tumors with infiltration exceeding $180^{\circ}$ of circumference showed a worse survival and more frequent presence of tumorous infiltration of the SMV/PV wall requiring additional resection than tumors of a lesser degree [23]. In our work, we studied survival only for unambiguously resectable tumors. We found a noticeably influential trend on survival depending on the degree of the tumor interface with the SMV/PV wall found on the pre-operation CT. However, the results were not statistically significant.

The limiting factor of our cohort is its size. We strived to enroll only patients with clearly resectable tumors, who had a standard post-operation course and treatment to eliminate other impacts on the length of survival after surgery. According to our results, the group of resectable pancreatic tumors appears not to be a homogenous one. Patients had a worse survival length prognosis after the operation with an increasing interface between the tumor and the SMV/ PV wall, but there is no significant relationship between the tumor interface with the vein wall and the incidence of R1 resections. Other described CT-indicated tumor characteristics (size and tumor margin degree) affected neither the incidence of R1 resections nor the length of survival.

In conclusion, the effort to achieve an $\mathrm{R} 0$ resection is the principal rule in the surgical treatment of PDAC. The current quality of the imaging methods does not allow for the possibility of an accurate prediction of R0 resection. In our cohort of patients assessed as resectable upon the initial CT, an R1 resection was achieved in $79.7 \%$ of cases. In $27 \%$ of the R1 resections, we found one positive resection line while in $73 \%$, two and more resection lines were positive. No difference was found in the incidence of $\mathrm{R} 1$ resections based on tumor characteristics determined on the CT: interface with SMV/PV wall, margin degree, and tumor size. A difference was found in survival after the operation depending on the tumor interface with the vein wall found on the CT, with the longest survival detected in patients having no interface between the tumor and the vein wall. However, the result was not statistically significant. It is, therefore, possible that PDAC of the head, currently graded as clearly resectable, is not a homogenous group and the patients differ in the prognosis of their disease.

Acknowledgments: Supported by Ministry of Health, Czech Republic - conceptual development of research organization (FNBr, 65269705).

\section{References}

[1] BRAY F., FERLAY J., SOERJOMATARAM I., SIEGEL RL, TORREBRAY LA et al. Global cancer statistics 2018: GLOBOCAN estimates of incidence and mortality worldwide for 36 cancers in 185 countries. CA Cancer J Clin 2018; 68: 394-424. https://doi.org/10.3322/caac.21492
[2] GHANEH P, COSTELLO E, NEOPTOLEMOS JP. Biology and management of pancreatic cancer. Gut 2007; 56: 11341152. https://doi.org/10.1136/gut.2006.103333

[3] LI D, XIE K, WOLFF R, ABBRUZZESE JL. Pancreatic cancer. Lancet 2004; 363: 1049-1057. https://doi.org/10.1016/ S0140-6736(04)15841-8

[4] ALLEMA JH, REINDERS ME, VAN GULIK TM, VAN LEEUWEN DJ, DE WITET LT et al. Portal vein resection in patients undergoing pancreatoduodenectomy for carcinoma of the pancreatic head. Br J Surg 1994; 81: 1642-1646. https://doi.org/10.1002/bjs.1800811126

[5] TSENG JF, RAUT CP, LEE JE, PISTERS PWT, VAUTHEYET JN et al. Pancreaticoduodenectomy with vascular resection: margin status and survival duration. J Gastrointest Surg 2004; 8: 935-949; discussion 949-950. https://doi. org/10.1016/j.gassur.2004.09.046

[6] KATZ MHG, MARSH R, HERMAN JM, SHI Q, COLLISON E et al. Borderline resectable pancreatic cancer: need for standardization and methods for optimal clinical trial design. Ann Surg Oncol 2013; 20: 2787-2795. https://doi. org/10.1245/s10434-013-2886-9

[7] CALLERY MP, CHANG KJ, FISHMAN EK, TALAMONTI MS, TRAVERSO LW et al. Pretreatment assessment of resectable pancreatic cancer: expert consensus statement. Ann Surg Oncol 2009; 16: 1727-1733. https://doi.org/10.1245/ s10434-009-0408-6

[8] EVANS DB, ERICKSON BA, RITCH P. Borderline resectable pancreatic cancer: definitions and the importance of multimodal therapy. Ann Surg Oncol 2010; 17: 2803-2805. https://doi.org/10.1245/s10434-010-1285-8

[9] SOHN TA, YEO CJ, CAMERON JL, KONIARIS L, KAUSHALET $S$ et al. Resected adenocarcinoma of the pancreas 616 patients: results, outcomes and prognostics indicators. J Gastrointest Surg 2000; 4: 567-579. https://doi.org/10.1016/ s1091-255x(00)80105-5

[10] NEOPTOLEMOS JP, STOCKEN DD, DUNN JA, ALMOND J, BEGER HG et al. Influence of resection margins on survival for patiens with pancreatic cancer treated by adjuvant chemoradiation and/or chemotherapy in the ESPAC-1 randomized controlled trial. Ann Surg 2001; 234: 758-768. https://doi.org/10.1097/00000658-20011200000007

[11] VERBEKE CS, LEITCH D, MENON KV, MCMAHON MJ, GUILLOUET PJ et al. Redefining the R1 resection in pancreatic cancer. Br J Surg 2006; 93: 1232-1237. https://doi. org/10.1002/bjs.5397

[12] ISHIKAWA O, OHIGASHI H, IMAOKA S, FURUKAWA $\mathrm{H}$, SASAKI $\mathrm{Y}$ et al. Preoperative indications for extended pancreatectomy for locally advanced pancreas cancer involving the portal vein. Ann Surg 1992; 215: 231-236. https://doi. org/10.1097/00000658-199203000-00006

[13] GIOVINAZZO F, TURRI G, KATZ MH, HEATON N, AHMED I et al. Meta-analysis of benefits of portal-superior mesenteric vein resection in pancreatic resection for ductal adenocarcinoma. Br J Surg 2016; 103: 179-191. https://doi. org/10.1002/bjs.9969 
[14] RAUT CP, TSENG JF, SUN CC, WANG H, WOLFF RA et al. Impact of resection status on pattern of failure and survival after pancreaticoduodenectomy for pancreatic adenocarcinoma. Ann Surg 2007; 246: 52-60. https://doi. org/10.1097/01.sla.0000259391.84304.2b

[15] MATHUR A, ROSS SB, LUBERICE K, KURIAN T, VICE M et al. Margin status impacts survival after pancreaticoduodenectomy but negative margins should not be pursued. Am Surg 2014; 80: 353-360.

[16] DIKMEN K, KEREM M, BOSTANCI H, SARE M, EKINCI $\mathrm{O}$ et al. Intra-Operative Frozen Section Histology of the Pancreatic Resection Margins and Clinical Outcome of Patients with Adenocarcinoma of the Head of the Pancreas Undergoing Pancreaticoduodenectomy. Med Sci Monit 2018; 24: 4905-4913. https://doi.org/10.12659/MSM.910279

[17] JAMIESON NB, FOULIS AK, OIEN KA, GOING JJ, GLEN $\mathrm{P}$ et al. Positive mobilisation margins alone do not influence survival following pancreatico-duodenectomy for pancreatic ductal adenocarcinoma. Ann Surg 2010; 251: 1003-1010. https://doi.org/10.1097/SLA.0b013e3181d77369

[18] DELPERO JR, BACHELLIER P, REGENET N, LE TREUT YP, PAYE F et al. Pancreaticoduodenectomy for pancreatic ductal adenocarcinoma: a French multicenter prospective evaluation of resection margins in 150 evaluable specimen. HPB (Oxford) 2014; 16: 20-33. https://doi.org/10.1111/ hpb.12061
[19] HOHENBERGER W, WEBER K, MATZEL K, PAPADOPOULOS T, MERKEL $S$ et al. Standardized surgery for colonic cancer: complete mesocolic excision and central ligation - technical notes and outcome. Colorectal Dis 2009; 11: 354-364; discussion 364-365. https://doi.org/10.1111/ j.1463-1318.2008.01735.x

[20] QUIROS RM, BROWN KM, HOFFMAN JP. Neoadjuvant therapy in pancreatic cancer. Cancer Invest 2007; 25: 267273. https://doi.org/10.1080/07357900701206356

[21] CHANDRASEGARAM MD, GOLDSTEIN D, SIMES J, GEBSKI V, KENCH JG et al. Meta-analysis of radical resection rates and margin assessment in pancreatic cancer. Br J Surg 2015; 102: 1459-1472. https://doi.org/10.1002/bjs.9892

[22] HARTWIG W, HACKERT T, HINZ U, GLUTH A, BERGMANN F et al. Pancreatic cancer surgery in the new millennium: better prediction of outcome. Ann Surg 2011; 254: 311-319. https://doi.org/10.1097/SLA.0b013e31821fd334

[23] CAO HT, BALACHRAN A, WANG H, NOGUERASGONZÁLEZ GM, BAILEY CE et al. Radiographic tumorvein interface as a predictor of intraoperative, pathologic and oncologic outcomes in resectable and borderline resectable pancreatic cancer. J Gastrointest Surg 2014; 18: 269-278. https://doi.org/10.1007/s11605-013-2374-3 